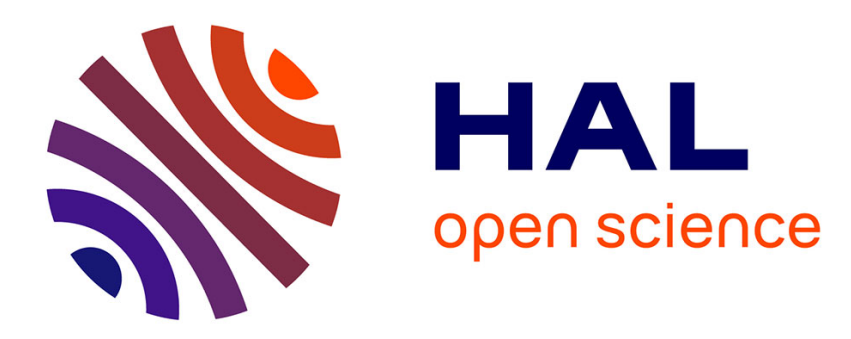

\title{
L'ancrage territorial d'une filière fromagère d'AOC. L'exemple du système Roquefort
}

Julien Frayssignes

\section{To cite this version:}

Julien Frayssignes. L'ancrage territorial d'une filière fromagère d'AOC. L'exemple du système Roquefort. Economie Rurale, 2001, 264-265, pp.89 - 103. 10.3406/ecoru.2001.5259 . hal-01564582

\section{HAL Id: hal-01564582 \\ https://hal.science/hal-01564582}

Submitted on 18 Jul 2017

HAL is a multi-disciplinary open access archive for the deposit and dissemination of scientific research documents, whether they are published or not. The documents may come from teaching and research institutions in France or abroad, or from public or private research centers.
L'archive ouverte pluridisciplinaire HAL, est destinée au dépôt et à la diffusion de documents scientifiques de niveau recherche, publiés ou non, émanant des établissements d'enseignement et de recherche français ou étrangers, des laboratoires publics ou privés. 


\section{L'ancrage territorial d'une filière fromagère d'AOC. L'exemple du système Roquefort \\ M Julien Frayssignes}

\section{Abstract}

The territorial footing of an aoc cheese industry: the example of the roquefort system - This article intends to study the evolution of the relationships between an old cheese industry - Roquefort - and its territory. In spite of deeply divergent logics and recurrent crisis, the economic and institutional pereniality of this system is a reality. In accordance with us, the territorial footing of the industry is a foundation of its pereniality, so, this complex notion must be clarified, notably in order to highlight its numerous dimensions.

\section{Résumé}

Cet article se propose d'étudier l'évolution du rapport au territoire d'une filière fromagère ancienne: Roquefort. Malgré des logiques profondément divergentes et des crises récurrentes, la pérennité économique et institutionnelle de ce système productif est une réalité, bancrage territorial de la filière étant un fondement de cette pérennité, il convient d'expliciter cette notion complexe en essayant de faire apparaître ses nombreuses dimensions.

\section{Citer ce document / Cite this document :}

Frayssignes Julien. L'ancrage territorial d'une filière fromagère d'AOC. L'exemple du système Roquefort. In: Économie rurale. N²64-265, 2001. L'agro-alimentaire dans la globalisation Entreprises, marchés, terroirs. pp. 89-103;

doi : 10.3406/ecoru.2001.5259

http://www.persee.fr/doc/ecoru_0013-0559_2001_num_264_1_5259

Document généré le 16/06/2016 


\section{ancrage territorial d'une filière fromagère d'AOC L'exemple du système Roquefort}

Julien FRAYSSIGNES • École nationale supérieure agronomique, Institut national polytechnique, UMR Dynamiques rurales, Toulouse

En France, les Signes officiels de qualité (SOQ) sont nés sous l'impulsion conjuguée des pouvoirs publics - dont le but était de limiter la baisse des revenus agricoles dans les régions dites difficiles - et des acteurs locaux, désireux de protéger et de valoriser des productions menacées de disparition. Fondées sur un mode de fonctionnement spécifique (différenciation par l'origine), les filières fabriquant et commercialisant des produits sous une Appellation d'origine contrôlée (AOC) ont été en mesure de bien résister aux évolutions quantitatives et qualitatives qui ont touché les secteurs agricole et agro-alimentaire durant ces quarante dernières années.

Néanmoins, aujourd'hui encore, ces filières sont constamment menacées par un environnement politique, économique et social incertain et désormais global. La crise de confiance des consommateurs, due à des problèmes sanitaires et à leur mauvaise gestion, et les négociations commerciales dans le cadre de l'OMC sont à cet égard deux défis majeurs pour la capacité de reproduction de ces systèmes productifs. Ces problèmes sont d'ailleurs particulièrement cruciaux si l'on considère les $A O C$ anciennes qui, du fait de leur notoriété, ont acquis une place privilégiée auprès des consommateurs.

La filière Roquefort ${ }^{1}$, située dans le sud du Massif Central, constitue de ce point de vue un objet d'étude tout à fait intéressant pour analyser les interrelations entre dynamiques locales et évolutions globales. En effet, outre les producteurs de lait, cette filière à la particularité de regrouper, au sein d'une organisation interprofessionnelle fortement structurée, une grande entreprise (la Société des Caves ou plus simplement Société), ellemême détenue par un grand groupe industriel français (Lactalis), et des unités de taille beaucoup plus réduite (PME, coopératives et maisons familiales).

L'un des caractères fondamentaux de cette organisation complexe est le fait que, malgré cette diversité, la filière soit parvenue, depuis sa création institutionnelle dans les années 1930, à maintenir sa cohérence et à assurer sa reproduction. La question qui se pose alors est la suivante: quels sont les facteurs de cette cohérence? Comment les acteurs de la filière sont-ils jusqu'à présent toujours parvenus à des compromis permettant à l'ensemble du système de conserver son unité?

Cet article pose l'hypothèse que les différentes formes de rapport au territoire de la filière sont un facteur d'explication décisif de sa reproduction institutionnelle et, par-là même, de sa performance économique. Plus vaste que le simple ancrage au terroir, l'examen de l'ancrage au territoire de la filière et

1. L'expression «filière Roquefort» désigne en fait un système productif dont l'activité dépasse la simple production du fromage AOC. En 1999, sur 170 millions de litres de lait collectés, 90 millions ont servi à la production de Roquefort, le reste étant soit exporté, soit transformé en féta, en pâtes pressées ou en différentes spécialités telles que le pérail. 
des acteurs, qui la composent, se révèle nécessaire afin d'appréhender les capacités du système à articuler logiques économiques et logiques territoriales.

Le questionnement exposé ici entend dépasser l'approche spatiale fondée sur la simple localisation des activités, où les entreprises s'implanteraient dans un espace en fonction du coût de transport ou de critères plus qualitatifs (présence d'infrastructures, fiscalité, proximité du marché, compétences...). Nous nous appuyons davantage sur les travaux de Jean-Benoît Zimmermann, pour qui l'ancrage territorial correspond à un «processus d'apprentissage collectif localisé et orienté vers la création de ressources» (Zimmermann, 1998).

Toutefois, si l'ancrage territorial tel que nous l'envisageons peut effectivement s'appréhender comme la mise en cohérence de stratégies divergentes, une hypothèse souvent inhérente à ces travaux est de le considérer comme un choix stratégique pour l'entreprise, qui décide - ou non - de mobiliser les ressources au sens large du territoire concerné. Cette option méthodologique peut s'apparenter à un certain «possibilisme» où le territoire propose et l'entreprise dispose ${ }^{2}$.

Selon nous, dans le cadre d'une $A O C$, et qui plus est d'une $A O C$ ancienne comme Roquefort, l'ancrage au territoire n'est pas toujours un choix effectué de manière consciente et intentionnelle. Il s'agit également d'un état de fait, d'une contrainte, que les acteurs - en particulier les industriels - ne peuvent maîtriser entièrement.

2. En géographie, le possibilisme est une attitude scientifique qui consistait à supposer que la nature offre un certain éventail de «possibilités» entre lesquelles l'homme pouvait choisir. La marge de manœuvre d'une société donnée était donc en grande partie dépendante de son avancement technique et de ses capacités d'organisation. Des auteurs tels que Paul Vidal de la Blache (1845-1918), sans toutefois jamais revendiquer cette appellation, ont été qualifiés de possibilistes.
Dans une première partie, nous essaierons de montrer qu'en dépit des acteurs aux stratégies divergentes et un environnement fluctuant, la filière parvient à se maintenir et à assurer sa reproduction économique et institutionnelle. Cette pérennité est selon nous en partie imputable aux caractéristiques du territoire dans lequel elle est inscrite. Nous nous attacherons dans une deuxième partie à proposer une grille de lecture destinée à formaliser les différentes formes d'ancrage territorial et à expliquer leur rôle dans la trajectoire du système.

\section{Reproduction du système et gestion des crises internes et externes}

L'AOC Roquefort, obtenue en 1925, est la plus ancienne appellation d'origine fromagère. En outre, elle est la seule à avoir fait l'objet d'une loi et non d'un décret. L'interprofession, baptisée Confédération de Roquefort, a été créée en 1930. Née de la fusion entre la fédération des industriels et la fédération des éleveurs, elle regroupe l'ensemble de ces acteurs dans le cadre d'une seule et même organisation.

Selon l'INAO, le Roquefort, fabriqué exclusivement avec du lait de brebis, représentait en 1999 la troisième $A O C$ fromagère en termes de tonnage derrière le Comté et le Cantal $^{3}$. En outre, avec environ 3000 tonnes durant l'année 1999, il s'agissait de la première AOC exportatrice. Aujourd'hui, l'aire de collecte du lait englobe un peu moins de 2500 exploitations agricoles et s'étend sur le sud de l'Aveyron, ainsi que sur une partie du Tarn, de la Lozère, de l'Hérault, du Gard, de l'Aude et du Tarn-et-Garonne. Les emplois industriels représentent environ 1700 personnes. L'importance du poids économique régional de l'activité liée à Roquefort est un autre caractère essentiel de la filière: environ

3. Comté: 40600 t; Cantal: 18300 t; Roquefort: 17600 t. (source: INAO, 1999). 
$50 \%$ de la valeur ajoutée et plus de $45 \%$ des emplois industriels du sud-Aveyron (arrondissement de Millau). Si l'on inclut les emplois induits (transporteurs, imprimeurs...), l'activité fromagère roquefortaise représente alors plus de 10000 actifs, et constitue ainsi l'acteur économique majeur de cette région (Guiraud, 1997).

Depuis son émergence institutionnelle dans les années 1925-1930, le système fait preuve d'une remarquable capacité de résistance aux crises auxquelles il est confronté. Ces crises sont aussi bien dues à des stratégies internes divergentes qu'à un environnement économique, social et politique en perpétuelle évolution.

\section{Les facteurs d'instabilité: types d'acteurs et environnement extérieur}

Si l'on considère le stade de la transformation, le processus de concentration industrielle est ininterrompu depuis une trentaine d'années. De 18 entreprises en 1970, on passe à 12 en 1975 et à 7 aujourd'hui. Malgré tout, la diversité des intervenants en aval de la filière demeure une réalité. Le groupe Société des Caves, détenı majoritairement par Lactalis, contrôle environ les deux tiers de la production. Le tiers restant est assuré par six autres entreprises: trois PME dont une coopérative, et trois maisons familiales. La domination de Société est d'ailleurs ancienne puisque dès 1925 , l'entreprise représentait déjà environ $60 \%$ de la production de Roquefort.

Du fait de cette structure industrielle originale, le système Roquefort a du mal à entrer dans les typologies élaborées par certains économistes dans le but d'analyser les dynamiques à l'œuvre au sein des AOC fromagères.

Afin de rendre compte de la très grande diversité des situations rencontrées sur le terrain, Philippe Perrier-Cornet et Bertil Sylvander proposent une typologie des $A O C$ fromagères à travers la confrontation entre logiques sectorielles et logiques territoriales
(Perrier-Cornet, Sylvander, 2000). Ils parviennent dès lors à identifier quatre formes de coopérations entre acteurs, appelées aussi gouvernances:

- Une «gouvernance sectorielle pure», où les coordinations dépendent avant tout d'un domaine d'activité et de ses normes spécifiques (pas de réel objectif $A O C$, pas de moyen de gestion en commun).

- Une «gouvernance sectorielle $A O C$ », où la valorisation du produit (sous des formes parfuis différenciées) conditionne les relations entre les acteurs.

- Une «gouvernance territoriale forte» et une «gouvernance territoriale faible», où le maintien et le développement des activités de la zone sont des objectifs plus ou moins essentiels.

Situer le système Roquefort au sein de cet éventail se révèle délicat. Si l'on considère les horizons sectoriels et territoriaux des acteurs de la filière, la préoccupation principale demeure bel et bien le produit et sa valorisation (gouvernance sectorielle AOC). Cependant, les enjeux territoriaux (développement agricole et rural, maintien des emplois et de la population) sont eux aussi tout à fait cruciaux et conditionnent cn partic lc comportement des acteurs. Il semble donc avéré que des formes de gouvernance «composites» existent, ce qui tend à nuancer l'opposition classique entre secteur et territoire.

Quoi qu'il en soit, cette diversité des transformateurs au sein de la filière induit une diversité des stratégies. L'arrivée du groupe Lactalis $^{4}$ (ex-Besnier) dans le capital de la Société des Caves en 1992 est à l'origine d'évolutions significatives de l'organisation et du fonctionnement de l'entreprise (stratégies industrielle et commerciale, intensification de la politique de diversification...).

4. En 1970, suite à des difficultés financières, Société fait l'objet d'une prise de participation par le groupe Perrier. Puis, en 1991, Perrier est à son tour racheté par le groupe suisse Nestlé, qui décide de remettre en vente ses parts de la Société des Caves, acquises par Lactalis l'année suivante. 
Pour Lactalis, déjà présent dans de nombreuses autres AOC fromagères, l'importance de l'entreprise aveyronnaise mériterait assurément une étude plus approfondie. Néanmoins, il semble que ce soit d'abord sa valeur patrimoniale et historique qui ait attiré le groupe de Laval. Si les pressions envers les autres entreprises sont réelles ${ }^{5}$, l'obtention d'un monopole total ne paraît pas être le but recherché. À travers sa présence, Lactalis semble avant tout vouloir «occuper le tcrrain», ct ćvitcr ainsi l'irruption de groupes industriels concurrents. En revanche, au niveau de l'interprofession, il n'est pas certain que les changements auraient été aussi importants. Le groupe paraît en fait vouloir laisser au système son autonomie de fonctionnement (notamment au niveau de la collecte et de la transformation) et récupérer à la sortie un produit à haute valeur ajoutée. Daniel Ricard note d'ailleurs le même type de comportement de la part des grands groupes (dont Lactalis) dans les filières AOC Reblochon et Comté (Ricard, 1993).

La stratégie de Lactalis à travers la Société des Caves contraste bien évidemment avec celle des autres entreprises. Si l'AOC Roquefort n'cst qu'un volct parmi d'autres dans la stratégie globale du groupe industriel (à travers la mise en place d'une gamme élargie de produits à base de lait de brebis), le but des maisons familiales par exemple est avant tout de se maintenir, notamment en recherchant des niches de marché spécifiques ${ }^{6}$.

L'entreprise SCARO (Société coopérative agricole régionale ovine) occupe du fait de son statut et de son histoire une place à part au sein de l'interprofession. Créée en 1976 sur l'initiative d'éleveurs aveyronnais et tar-

5. La politique de croissance externe menée par Société est en effet susceptible de représenter une menace pour les autres entreprises. En 1997, une entreprise familiale, Crouzat, a d'ailleurs été rachetée et intégrée au capital de Société.

6. Les établissements Vernières, qui assuraient environ $3 \%$ du tonnage AOC en 1999, exportent plus du tiers de leur production aux États-Unis. nais, la SCARO, rachetée depuis par le groupe coopératif 3A (Alliance Agro-Alimentaire), constitue aujourd'hui la deuxième entreprise produisant le fromage de Roquefort.

En 1987, confrontés à une surproduction laitière croissante, les dirigeants de la filière décident, outre la diversification, de mettre en place un système original de paiement du lait: les Volumes individuels de référence $(\mathrm{VIR})^{7}$. Les réactions négatives seront nombreuses, notamment de la part d'éleveurs qui fonderont en 1991 le Syndicat des producteurs de lait de brebis (SPLB). C'est toutefois la SCARO qui va cristalliser les oppositions à ce nouveau système de paiement du lait jugé inégalitaire ${ }^{8}$. Dès 1988 , la coopérative décide de se retirer de l'interprofession et de payer son lait à un prix unique. Après d'âpres négociations, SCARO et Confédération parviendront finalement à un accord et en 1994, la coopérative réintégrera l'interprofession au prix de quelques aménagements. La crise occasionnée par cette «sécession» aura été l'une des plus graves vécue par la Confédération de Roquefort, l'hypothèse de sa disparition ayant même été évoquée. En effet, dans un système de collecte intégrale de la matière première et de prix de revient unique pour les industriels, c'est l'ensemble du marché du fromage de Roquefort qui a été déstabilisé par la dissidence de la SCARO.

$\mathrm{Si}$ les crises «internes», inhérentes au comportement des acteurs, ont constamment menacé la fragile cohésion du système, ce-

7. Dès l'annéc suivante, chaque producteur se voit attribuer un volume à produire calculé sur la base des quatre dernières campagnes (1983 à 1987) et correspondant à la quantité de lait devant être transformé en Roquefort (environ 7 F/litre). Contrairement aux quotas chez les bovins, le dépassement de la référence n'est pas sanctionné, le lait en trop est alors destiné à la diversification (lait en poudre, féta...) et fait l'objet d'une rémunération moins élevée.

8. Du fait de son statut coopératif, la SCARO entendait conserver le pouvoir de déterminer elle-même le prix du lait acheté aux producteurs, indépendamment de tout système imposé par la Confédération. 
lui-ci a également dû faire face aux sollicitations de son environnement extérieur, notamment économique et politique.

L'histoire de la filière est en effet jalonnée d'épisodes ayant eu comme conséquence une remise en cause partielle de son fonctionnement et de sa légitimité. Le conflit avec la Grèce au sujet de la dénomination féta a constitué pour la filière un enjeu de premier plan ${ }^{9}$. Revendiqué par les Grecs, le terme féta a finalement été reconnu comme générique par l'Union européenne (car fabriquée partout sur le continent), ce qui a évité à la filière dans son ensemble des préjudices financiers importants. On peut également citer la décision de la part des États-Unis de surtaxer certains produits de luxe européens (dont le Roquefort), suite à la décision de Bruxelles d'interdire l'importation de bœuf américain aux hormones.

Ces crises représentent de lourdes menaces pour la filière, en particulier du point de vue économique. Toutefois, leur origine exogène tend à réduire les divergences d'intérêt et à renforcer la cohésion des acteurs.

Ainsi, en dépit des crises internes et des menaces extérieures, le système roquefortais parvient à sc maintenir ct à assurer sa reproduction. À la manière du vignoble languedocien analysé par le géographe Franck Auriac, la filière Roquefort parvient de manière permanente à «surmonter ses contradictions internes et à résister de manière efficace aux évolutions extérieures" (Auriac, 1983). Dans une perspective résolument systémique, l'auteur met en évidence la relative cohérence du système viticole et sa capacité à minimiser son entropie ${ }^{10}$. Malgré une approche quelque peu «holiste» qui personnifie parfois de manière un peu trop caricaturale

9. La transformation en féta représente pour l'interprofession la première source de diversification avec environ $\mathbf{4 0}$ millions de litres transformés sur 170 en 1999 (numéro un au niveau national), pour un prix voisin de cinq francs payé à l'éleveur.

10. L'entropie d'un système social désigne son état de désordre, et donc l'absence de cohérence globale. l'objet de sa recherche, Auriac estime que c'est à travers son assise spatiale que le vignoble parvient à se reproduire. La filière Roquefort semble ainsi relever d'une problématique comparable, c'est pourquoi il nous semble nécessaire de réfléchir à présent sur les fondements de sa cohérence et sur le rôle joué par le territoire dans cette pérennité.

\section{La pérennité du système et ses fondements territoriaux}

D'un point de vue systémique, le terme de pérennité renvoie d'abord à l'idée de durée, de permanence, et par-là même de temporalité. La pérennité d'un système désigne sa capacité de résistance à l'usure du temps, ceci à travers l'émergence de processus organisationnels permanents. Ainsi, la cohérence et l'autonomie sont deux variables fondamentales permettant d'appréhender la pérennité d'une organisation.

- La cohérence d'un système désigne l'absence (relative) de contradiction, les éléments se coordonnent entre eux de manière logique et efficace pour parvenir à un objectif précis. Dans le cas de Roquefort, la cohérence du système se manifeste à travers la gestion des conflits et des crises auxquels nous avons fait allusion plus haut. Depuis sa mise en place dans les années 1930, l'interprofession roquefortaise a constitué le seul et unique espace de négociation pour les acteurs de la filière. Les décisions inhérentes aux destinées de l'organisation ont toujours été prises dans ce cadre, ce qui tend à prouver son efficience.

- L'autonomie se réfère quant à elle à la capacité organisationnelle. Un système est dit autonome lorsqu'il a la capacité de se gouverner selon des principes qui lui sont propres. Dans le cas des systèmes sociaux, l'autonomie n'est jamais totale, elle est toujours relative à un contexte extérieur qui lui impose des contraintes plus ou moins fortes. La Confédération a ainsi toujours été en mesure de conserver une marge de manœuvre 
vis-à-vis de son environnement. Cette propension à se fixer ses propres règles de fonctionnement s'est manifestée notamment à travers la mise en place des volumes de référence en 1987, système de paiement du lait unique et sans véritable équivalent dans les autres filières AOC françaises.

D'une manière générale, les règles institutionnelles qui organisent le système et les relations entre les acteurs se révèlent être d'une extrême complexité. Depuis la création de l'interprofession, aucune réforme en profondeur n'est venue simplifier les nombreux enchevêtrements juridiques. Le système a au contraire évolué par amendements successifs et de manière relativement autonome, les volumes de référence constituant en quelque sorte l'apothéose de la complexi$t e^{-11}$. Malgré une certaine rigidité, la filière n'est pas pour autant figée, chaque crise a eu au contraire comme incidence une évolution de l'ensemble de l'organisation.

En parallèle à ce dispositif juridique, le fonctionnement du système se caractérise par une série de règles informelles, de pratiques souvent fondées sur des accords anciens mais qui perdurent encore aujourd'hui sans jamais avoir été remises en cause de manière explicite. Une illustration frappante de ce fonctionnement informel nous est donnée par la gestion particulière de la matière première au niveau des entreprises de transformation. Lorsqu'un industriel vient à manquer de lait, il a en effet la possibilité de s'approvisionner auprès d'un autre industriel si celui-ci dispose de matière première en trop. Cette coopération est rendue possible par le fait qu'il n'existe pas de relation officiellement contractualisée entre industriels et producteurs de lait, ce qui autorise une relative souplesse.

11. Du point de vue de l'éleveur, le lait payé au prix maximum ne sera pas forcément transformé intégralement en Roquefort. I a valorisation se réalise au niveau global et avant tout en fonction des débouchés. Le système de paiement de la matière première n'est donc que théorique.
Ainsi, il semble exister - toutes proportions gardées - une réelle solidarité entre les acteurs de la filière. Cette conscience collective est à rapprocher de la notion de confiance organisationnelle, développée notamment par Eduardo Chia et André Torre. Dans un article consacré à la filière Comté, ces deux auteurs définissent la confiance comme une "attente mutuelle qu'un individu n'exploitera pas les espaces de vulnérabilité ouverts par les relations de coopération" (Chia, Torre, 1999). La confiance organisationnelle peut quant à elle se définir comme un apprentissage d'engagements mutuels structuré par la fixation de règles prédéfinies. Elle transcende ainsi en partie les intérêts individuels, notamment si la filière est confrontée à des évolutions de son environnement. Malgré leur statut de simples fournisseurs de matière première, les éleveurs de brebis se considèrent comme membres de l'interprofession à part entière, et revendiquent clairement leur rôle de garant de la qualité du lait.

Si la conscience d'une destinée commune est palpable lorsque l'on considère les enjeux extérieurs, c'est aussi et surtout dans les négociations internes que se manifeste la cohésion du système. En dépit de la diversité des intervenants, la filière est parvenue à surmonter de nombreux paradoxes grâce à une véritable «culture de la concertation», culture dont l'origine semble avant tout être imputable aux acteurs locaux ${ }^{12}$. La Confédération de Roquefort apparaît ainsi comme un organisme de gestion des crises à travers l'instauration de compromis durables.

Élément d'explication déterminant dans la reproduction institutionnelle et la réussite économique de la filière, le mode de fonc-

12. Les acteurs de la filière ont par exemple pris conscience très tôt de l'enjeu que représentait la présence de listéria dans les fromages au lait cru. Des contrôles quotidiens du lait ont donc été mis en place de manière spontanée dès 1990. La capacité organisationnelle du système se double ainsi de ce que l'on pourrait appeler une précocité organisationnelle. 
tionnement original du système Roquefort est en partie imputable aux caractères socioculturels du territoire dans lequel il est inscrit. Le village de Roquefort, lieu où le fromage est affiné, est situé près de Millau, dans le sud du département de l'Aveyron. Il s'agit d'un territoire à l'identité très marquée, notamment vis-à-vis du reste du département, resté célèbre en raison de la contestation paysanne dans les années 1970, en réaction au projet d'agrandissement du camp militaire du Larzac ${ }^{13}$.

Si la filière Roquefort constitue un élément fortement structurant pour le territoire, aussi bien en termes d'emploi que d'occupation de l'espace et de notoriété, le territoire exerce en retour une influence forte sur les acteurs de la filière. L'attachement des acteurs - producteurs de lait et industriels - à leur territoire d'origine se manifeste en permanence et influe de manière significative sur l'organisation de la filière. Lors de la prise de participation de Perrier en 1970, les dirigeants des éleveurs, craignant une perte de pouvoir de décision au niveau local, ont créé avec la Caisse régionale de crédit agricole la Sodagri (Société de développement agricole) et ont ainsi pu racheter $25 \%$ du capital de la Société des Caves. La Sodagri demeure aujourd'hui encore le deuxième actionnaire de l'entreprise après Lactalis. Un élément frappant est le fait que cette prégnance locale s'exerce également sur les acteurs venus de l'extérieur, notamment en ce qui concerne les industriels. La stratégie industrielle et commerciale du groupe Lactalis semble en effet infléchie par de réelles préoccupations territoriales. Dans les discours et les pratiques des dirigeants, les notions de développement agricole et rural sont récurrentes ${ }^{14}$. Ce phénomène «d'acculturation" apparaît pour Lactalis comme un

13. C'est aussi à Millau qu'à eu lieu en juin 2000 le procès du syndicaliste José Bové et la grande fête populaire dénonçant les dérives de la mondialisation. point de passage obligé qui engage sa légitimité auprès des acteurs locaux.

Le territoire est donc ici un lieu de pouvoir structuré par des règles formelles et informelles qui contraignent fortement la logique productive du groupe. L'organisation de l'interprofession roquefortaise est d'un point de vue historique fortement imprégnée des caractères sociaux, économiques, politiques et culturels du territoire sud-aveyronnais. Ces multiples rapports avec le territoire apparaissent donc comme un des fondements de la permanence du système et de son adaptation à son environnement concurrentiel. Pour les acteurs de la filière, et notamment les acteurs extérieurs, l'ancrage territorial s'apparente certes à un choix stratégique, mais également à des contraintes avec lesquelles il leur faut compter ${ }^{15}$.

Les interpénétrations entre la structure et le fonctionnement de la filière et son territoire sont multiples. L'articulation entre pérennité et ancrage nécessite donc une formalisation dans le cadre d'une grille d'analyse que nous proposons de développer dans la deuxième partie.

\section{Pérennité et ancrage territorial une tentative d'explication}

Dans les travaux faisant état du comportement spatial des firmes, l'accent est mis sur la dualité entre «ancrage territorial» et «nomadisme» (Zimmermann, 1998). La no-

14. Le groupe Société semble depuis peu s'impliquer de manière significative dans la vie locale, notamment à travers la participation financière à des manifestations culturelles (Mondial de Pétanque de Millau, Festival de la brebis Lacaune). Cette pratique s'inscrit certes dans une perspective de communication, mais semble être quasi-obligatoire pour légitimer la présence du groupe dans la filière, et par là même dans le territoire.

15. Une phrase relevée dans un entretien avec un dirigeant de l'entreprise est à cet égard révélatrice, parlant de ses relations avec les autres acteurs de la filière, celui-ci estime que «l'on se prend au jeu sans s'en rendre compte». 
tion d'ancrage apparaît comme un processus d'apprentissage collectif localisé, orienté vers la création de ressources. La fondation d'une communauté de destins entre une entreprise et un territoire se réalise en un lieu et à une époque donnés, ceci en fonction de la trajectoire de la firme et de ses objectifs. Dans ce sens et même s'il faut nuancer cette idée, l'ancrage s'oppose généralement au nomadisme et à la recherche de flexibilité et de réversibilité. Toutefois, malgré une approche réciproque des relations entre firme et territoire, l'ancrage (ou le non-ancrage) demeure toujours une option stratégique intentionnelle et consciente. La grille d'analyse - nonexhaustive - relative au rapport au territoire d'une filière que nous proposons ici relève certes en partie de cette intentionnalité, mais est également composée d'indicateurs qui mettent en avant l'idée de contrainte.

Notre réflexion sur Roquefort nous a permis de dégager des critères forts rendant compte de l'ancrage territorial de la filière. Même si la spécificité de l'objet considéré peut apparaître comme un obstacle, c'est néanmoins dans une perspective de généralisation à l'ensemble des systèmes produisant et commercialisant des fromages sous une appellation d'origine que s'élabore notre modèle.

\section{Le modèle d'analyse: formes d'ancrage et niveaux de complexité}

Le rapport au territoire tel que nous le développons ici peut s'appréhender selon trois niveaux d'analyse distincts mais interdépendants: celui de l'acteur, de la filière et du produit. Les acteurs d'une filière AOC stricto sensu (c'est-à-dire réduite à l'organisation interprofessionnelle) sont soit des producteurs (laitiers ou fermiers), soit des transformateurs (privés ou coopératifs, artisans ou industriels). Ensuite, de nombreux choix stratégiques s'effectuant au niveau de l'interprofession, celle-ci constitue également un cadre d'analyse pertinent dans la mesure où elle conserve une relative autonomie de décision et une certaine rationalité. Enfin, il nous a semblé nécessaire d'avoir une approche en termes de produit, puisqu'une filière $A O C$ désigne le plus souvent une organisation productive valorisant de manière diversifiée la matière première nécessaire à son fonctionnement. La coexistence de produits $A O C$ et non-AOC au sein d'un même système pose en effet des questions cruciales en termes d'ancrage territorial.

À chaque niveau d'analyse sont associées différentes formes de rapport au territoire certes liées entre elles mais pas de manière mécanique. Sans prétendre à l'exhaustivité, nous distinguons trois grandes familles d'indicateurs d'ancrage territorial:

- Les modalités de mobilisation des ressources productives, qui concernent l'ensemble des fonctions de production de la filière et des acteurs qui la composent. Ces ressources sont prises ici au sens large, il s'agit aussi bien d'éléments matériels (matières premières) que d'éléments immatériels (compétences, services...). Dans l'analyse des moyens mobilisés - et de leur origine - pour assurer le fonctionnement de la filière, le but est notamment de mettre en évidence les déterminants territoriaux qui sous-tendent les stratégies à l'œuvre.

- Les relations avec les acteurs territoriaux, qui sont inscrits dans les sphères politiques (élus locaux), sociale (agents et organismes de développement, organismes professionnels, formation...), économique (autres secteurs tels que le tourisme).

- La valorisation des caractères du territoire dans la promotion des produits. Les stratégies d'incorporation d'aménités ${ }^{16}$ dans les produits agro-alimentaires constituent en

16. Le terme d'aménité est utilisé pour désigner un ou plusieurs caractères altractifs d'un territoire. Un savoir-faire ou un paysage peuvent être définis comme des aménités (Couzinet, Frayssignes, Pouzenc, Simonneaux, 2000). 
effet un indicateur fondamental de l'ancrage territorial.

Ces trois types de rapport au territoire peuvent s'appréhender en fonction des trois niveaux d'analyse évoqués plus haut. L'élaboration du modèle explicatif consiste donc à croiser ces deux types de critères. Toutefois, une réflexion en termes d'ancrage ne peut s'effectuer sans avoir au préalable déterminé pour la filière $A O C$ considérée un référentiel territorial pertinent. Même si cela ne doit pas exclure l'usage d'autres échelles d'analyse (un grand groupe industriel élabore sa stratégie d'abord au niveau global), l'élaboration d'un cadre territorial dans laquelle la filière se définit et définit son action est selon nous une condition indispensable à la mise en pla- ce du modèle d'analyse. L'aire d'appellation du produit est certes un support indispensable, mais en fonction des systèmes productifs étudiés, il apparaît pertinent d'intégrer d'autres facteurs afin d'appréhender l'ensemble des dynamiques territoriales (limites naturelles, administratives, économiques, historiques, culturelles, identitaires...). Dans le cas de Roquefort, l'aire d'appellation vient se superposer à des entités administratives diverses (arrondissement de Millau, parc naturel régional des Grands Causses, structures intercommunales, bassin d'activité...), ce qui tend à façonner un territoire aux limites plus ou moins floues mais doté d'une réelle existence pour les acteurs qui s'y trouvent.

\section{Le rapport au territoire des filières fromagères $d^{\prime} A O C$}

\begin{tabular}{|c|c|c|c|}
\hline \multirow[t]{2}{*}{ Niveaux d'analyse } & \multicolumn{3}{|c|}{ Formes de rapport au territoire } \\
\hline & $\begin{array}{l}\text { Modalités } \\
\text { de mobilisation } \\
\text { des ressources } \\
\text { productives }\end{array}$ & $\begin{array}{l}\text { Relations } \\
\text { avec les autres acteurs } \\
\text { territoriaux }\end{array}$ & $\begin{array}{l}\text { Valorisation des caractères } \\
\text { du territoire } \\
\text { dans la promotion } \\
\text { des produits }\end{array}$ \\
\hline \multicolumn{4}{|l|}{ ACTEUR* } \\
\hline Producteur laitier & $\begin{array}{l}\text { Relations avec fournisseurs } \\
\text { et services (appui technique, } \\
\text { crédit, engrais, semences, } \\
\text { aliments...) }\end{array}$ & $\begin{array}{l}\text { Diversification } \\
\text { (tourisme rural, } \\
\text { production fermière...) }\end{array}$ & $\begin{array}{l}\text { Mise en valeur de l'aspect } \\
\text { traditionnel et authentique } \\
\text { du produit ou du service }\end{array}$ \\
\hline Transformateur & $\begin{array}{l}\text { Relations avec fournisseurs, } \\
\text { services et clients } \\
\text { (embauche, transport, } \\
\text { distribution locale...) }\end{array}$ & $\begin{array}{l}\text { Participation à des projets } \\
\text { de développement, } \\
\text { sponsoring }\end{array}$ & $\begin{array}{l}\text { Incorporation d'aménités } \\
\text { dans le produit AOC } \\
\text { (savoir-faire, paysages...) } \\
\text { Stratégie de marketing pour } \\
\text { les produits génériques }\end{array}$ \\
\hline $\begin{array}{l}\text { INTERPROFESSION } \\
\text { Cohérence } \\
\text { Autonomie } \\
\text { Capacité } \\
\text { organisationnelle }\end{array}$ & $\begin{array}{l}\text { Relations producteurs-trans- } \\
\text { formateurs. } \\
\text { Relations clients, fournisseurs } \\
\text { et services. } \\
\text { Poids économique }\end{array}$ & $\begin{array}{l}\text { Implication dans des projets } \\
\text { de développement local. } \\
\text { Synergie avec d'autres } \\
\text { secteurs (tourisme...) }\end{array}$ & $\begin{array}{l}\text { Incorporation d'aménités } \\
\text { dans le produit AOC }\end{array}$ \\
\hline $\begin{array}{l}\text { PRODUIT } \\
\text { AOC } \\
\text { diversification }\end{array}$ & $\begin{array}{l}\text { Localisation des activités, } \\
\text { contraintes techniques } \\
\text { inhérentes à I'AOC, lien } \\
\text { au terroir, évolution } \\
\text { de l'aire d'appellation }\end{array}$ & $\begin{array}{l}\text { Utilisation de la notoriété } \\
\text { des produits } \\
\text { par les acteurs locaux }\end{array}$ & $\begin{array}{l}\text { Confrontation des modalités } \\
\text { de valorisation différenciées } \\
\text { (origine VS marque } \\
\text { commerciale et/ou marque } \\
\text { de distributeur) }\end{array}$ \\
\hline \multicolumn{4}{|c|}{$\begin{array}{l}\text { ^ Dans le cas de Roquefort, la distinction entre producteurs laitiers et transformateurs est suffisante puisque la produc- } \\
\text { tion fermière a été abandonnée dès les années 1920. L'étude d'une filière comme celle du Saint-Nectaire nécessiterait } \\
\text { d'établir un clivage entre production fermière et production laitière. }\end{array}$} \\
\hline
\end{tabular}


Une fois les acteurs identifiés, il s'agit de caractériser leur positionnement territorial (acteur enraciné, nomade, opportuniste...) à travers l'étude de leur trajectoire. L'attachement au lieu et le sentiment d'appartenance d'un acteur sont des dimensions de sa territorialité $^{17}$, dimensions qui conditionnent en partie ses pratiques et ses représentations.

Les producteurs laitiers mobilisent leurs facteurs de production auprès des industries d'amont (crédit, appui technique, intrants...). Néanmoins, du fait de leur intégration plus ou moins avancée aux unités de transformation, leur marge de manœuvre dans le choix des fournisseurs est souvent réduite. De nombreuses décisions se prennent alors au niveau de l'interprofession. La diversification vers le tourisme rural ou vers une production fermière peut être l'occasion de «sortir» du cadre productif de la filière. Dans cette hypothèse, le producteur peut être amené à mobiliser de nouvelles ressources pour caractériser les produits ou services concernés. Dans une étude consacrée à Roquefort, la géographe Laurence Barthe estime que du fait de la plus-value générée par l'activité laitière et fromagère, la filière $a$ inconsciemment joué le rôle "d'inhibiteur de projets de développement local», les acteurs locaux - notamment les producteurs - ayant eu tendance à se reposer sur leurs acquis (Bonniol, Saussol, 1995).

Dans le cas des transformateurs, la question des relations avec les clients et les fournisseurs se pose avec plus d'acuité. Des éléments tels que l'origine de l'embauche ou le choix des transporteurs rendent compte de l'ancrage productif d'une entreprise. Toutefois, là encore, cet ancrage peut être rendu obligatoire par des contraintes territoriales et aller à l'encontre d'une recherche de minimisation des coûts. Les dirigeants de la filière

17. La territorialité d'un acteur ou d'un groupe d'acteurs peut se définir ici comme le rapport individucl ou collectif à un espace considéré comme approprié (Brunet, Ferras, Théry, 1993).
Roquefort font depuis longtemps appel à des transporteurs locaux pour acheminer les fromages, l'arrivée de Lactalis en 1992 n'a pas modifié ces pratiques, ceci en dépit du fait que le groupe lavallois dispose de son propre service de transport. Ces options stratégiques négociées entre les acteurs locaux et les acteurs extérieurs peuvent s'apparenter à ce que Jean Saglio appelle des "échanges sous contrainte de préférence identitaire» (Saglio, 1991).

Les entreprises de transformation sont également amenées à dépasser leur fonction productive et à entretenir des rapports avec les autres acteurs du territoire. Ainsi, les relations avec la sphère politique constituent à cet égard un bon révélateur du positionnement territorial de la firme. Vis-à-vis des projets de développement par exemple, l'entreprise peut en fonction de sa trajectoire et de sa stratégie entrer en concurrence avec les élus locaux, développer des synergies ou bien encore se révéler indifférente. Enfin, au niveau de la promotion, la présence d'une $A O C$ induit un lien au territoire particulier. Les stratégies de valorisation sont très différentes selon les acteurs considérés. Une petite entreprise va d'abord mettre en évidence l'origine et le côté authentique et traditionnel du produit tandis qu'un grand groupe peut être davantage enclin à fonder sa communication sur une marque commerciale. Les campagnes publicitaires élaborées par la Confédération de Roquefort et par la Société des Caves insistent d'abord sur les conditions naturelles d'affinage et sur les savoir-faire. Il est d'ailleurs intéressant de noter que l'origine même du produit est peu mise en évidence, ce qui contraste avec des filières telles que l'Ossau-Iraty ou le Comté, où les origines (respectivement basques et jurassiennes) sont clairement revendiquées.

Les indicateurs évoqués au niveau des acteurs peuvent être en partie repris au niveau de la filière (relations clients-fournisseurs, relations avec les acteurs politiques, économiques, de développement, promotion du 
territoire...). La territorialité des acteurs se manifeste également dans leurs relations (personnalisées ou non) au sein de l'interprofession. L'articulation cohérente des différentes stratégies de mobilisation des ressources est une des conditions de la reproduction institutionnelle et économique du système. Par ailleurs, à travers des critères classiques tels que le nombre d'emplois, le chiffre d'affaires ou la valeur ajoutée, le poids économique de la filière sur son territoire de référence est un élément d'analyse qui permet d'évaluer sa marge de manœuvre et son influence. Avec plus de 10000 emplois directement ou indirectement liés à la production du fromage en 1997 (dont 5500 actifs agricoles), la filière Roquefort représente «l'acteur» économique majeur du sud-Aveyron et joue de ce fait un rôle prépondérant dans l'évolution du tissu socio-économique de la région.

Posé en termes d'ancrage, le rapport au territoire des produits (AOC et non-AOC dans l'hypothèse d'une diversification) issus de l'activité de la filière relève de la stratégie des acteurs.

En ce qui concerne le produit $\mathrm{AOC}$, les contraintes juridiques imposées - ou non par le décret sont le résultat de la confrontation entre des logiques territoriales (protection d'une appellation, d'une région...) et des logiques productives (volume de collecte, rentabilité...). Si la zone d'affinage du fromage est limitée au village de Roquefort-surSoulzon par le texte de loi, la localisation des unités de collecte et de transformation répond, quant à elle, à des impératifs de rationalisation de la production ${ }^{18}$. L'évolution de l'aire d'appellation est également un indicateur d'ancrage tout à fait fondamental dans la mesure où il traduit pour un produit donné

18. En 1905, on dénombrait dans la région plus de 1000 laiteries destinées à la production de Roquefort, 750 en 1930 et 225 en 1975 . Aujourd'hui, la production s'organise autour de moins d'une dizaine de centrales laitières. les variations dans les rapports de force entre les acteurs de la filière. La «géométrie variable» de l'AOC Roquefort est à cet égard significative. Au début du siècle, du fait des besoins croissants en lait, le périmètre de collecte s'est étendu à toute la région sud du Massif Central ainsi qu'en Corse et dans les Pyrénées. Avec les progrès techniques et la forte augmentation de la productivité, la filière a abandonné les bassins extérieurs et l'aire s'est progressivement recentrée sur son territoire d'origine.

Par ailleurs, dans la mesure où le produit AOC génère une rente, les acteurs du territoire (politiques ou économiques) peuvent dans la réalisation de leurs actions s'appuyer sur sa notoriété et profiter eux aussi de cette rente. L'on pense notamment à des synergies qui peuvent se mettre en place entre la filière et le secteur du tourisme. La trajectoire du système et la stratégie des acteurs qui le composent sont alors déterminantes. La filière Roquefort s'inscrit avant tout dans une logique sectorielle, la recherche d'une valorisation optimale de la matière première est un objectif constant. Néanmoins, depuis quelques années, des coopérations se mettent en place avec des acteurs locaux. La création par quelques agriculteurs d'un réseau - certes modeste - de «visites de fermes en pays de Roquefort», en collaboration avec la Confédération et le Parc naturel régional des Grands Causses, est un exemple de coopération. En parallèle, depuis quelques années, commencent à émerger de petites productions fermières ou artisanales qui jouent le jeu de l'ancrage au terroir et au territoire.

Au sein d'une filière $A O C$, la stratégie de diversification vers des produits génériques (féta, poudre de lait) ou spécifiques (marques commerciales) est de plus en plus fréquente. Insufflée le plus souvent par les grands groupes, elle permet au système de réguler sa production en développant (ou en créant) de nouveaux débouchés. La coexistence de différents types de produits est susceptible de provoquer des changements 
dans l'organisation - notamment spatiale de la production et dans les relations entre les acteurs. Dans une perspective d'ancrage, la diversification peut s'apparenter à un "éloignement vis-à-vis du territoire de référence» (Frayssignes, 1999), surtout si elle prend de l'ampleur. La filière joue alors désormais «sur plusieurs tableaux» (origine et/ou marque commerciale), ce qui lui permet une plus grande flexibilité. Aujourd'hui, rappelons-le, la transformation en Roquefort concerne seulement $55 \%$ de la collecte laitière. La production de féta ou de différentes spécialités locales telles que le pérail et la vente de lait en l'état ou en poudre constituent les autres modalités de valorisation. L'expression de "filière Roquefort» doit donc faire l'objet d'une utilisation prudente. Pourtant, même pour un produit désormais générique comme la féta, l'ancrage territorial n'est pas complètement absent puisque son origine méditerranéenne est mise en évidence, notamment dans le discours des acteurs.

\section{Les facteurs extérieurs de l'ancrage}

Ainsi, la confrontation entre les trois formes de rapport au territoire et les trois niveaux d'analyse fait apparaître un début de modèle d'analyse apte selon nous à rendre compte de la diversité des trajectoires et des modes d'ancrage territorial des filières fromagères bénéficiant d'une AOC.

Afin de compléter cette grille d'analyse, il nous faut insister ici sur une idée essentielle. Le processus d'ancrage d'un acteur d'une interprofession $A O C$ donnée dans un territoire de référence se réalise certes à une échelle avant tout locale. Toutefois, dans la mesure où ces filières sont des systèmes ouverts sur l'extérieur, il convient également de considérer l'ancrage territorial comme un processus inscrit dans un environnement - ou plutôt dans des environnements - qui exercent une prégnance réelle sur ces filières et qui constituent de ce fait des facteurs plus ou moins propices à leur ancrage. Ces facteurs, qui se manifestent selon des modalités très différentes, sont de deux natures distinctes: économique et institutionnelle.

- Au niveau économique, de très nombreux travaux font état du phénomène de mondialisation et de ses conséquences sur le rapport au territoire des acteurs économiques. Pour Olivier Crevoisier, la globalisation des activités et la concurrence accrue qui en résulte obligent les organisations économiques à plus d'ouverture et plus de flexibilité. La nécessaire adaptation des entreprises à ces nouvelles conditions les incite à tenter de réduire leur dépendance vis-à-vis des régions où elles sont inscrites afin d'accroître leur réversibilité. Au vu de cette idée, la mondialisation de l'économie contribue à l'effacement des spécificités territoriales et semble donc un facteur limitant pour l'ancrage des acteurs économiques. Néanmoins, les thèses fondées sur un développement territorialisé et différencié (type système industriel localisé) mettent au contraire l'accent sur le caractère irréductible de chaque région. La mondialisation serait alors l'occasion pour les acteurs de mettre en place de nouvelles stratégies et de valoriser les ressources lociales. L'ancrage territorial des firmes s'en trouverait alors conforté (Proulx, 1998). Dans le cas plus spécifique des filières AOC, l'environnement économique se manifeste notamment à travers deux facteurs: la demande et la concurrence.

L'état de la demande constitue un élément d'explication essentiel. Les fromages bénéficiant d'une AOC sont certes positionnés sur des marchés garantis et rentables mais peu extensibles, ce qui tend à limiter leur croissance en termes de parts de marché. Ainsi, lorsque cela est possible, le recours à l'exportation peut alors se révéler être une bonne stratégie complémentaire. En outre, si la forte demande actuelle en matière de qualité alimentaire et de traçabilité joue plutôt en faveur des produits $A O C$, les récents accidents sanitaires qui ont touché le secteur fro- 
mager (listéria) posent à nouveau - à travers la maîtrise d'une matière première fragile (le lait) et son utilisation dans la fabrication des fromages d'appellation - la question de la pertinence de l'outil $A O C$ pour résoudre à lui seul ces problèmes.

À travers le lien au terroir, la typicité est un élément essentiel de la communication réalisée par les filières autour des produits bénéficiant d'une appellation. Parallèlement à ce travail, une réflexion menée sur les filières avicoles label rouge Avigers et les filières fromagères AOC Rocamadour et Roquefort nous montre qu'au-delà du simple terroir, les acteurs des interprofessions ont tout intérêt à se référer au territoire dans son ensemble. Ainsi, le poids d'une filière en termes d'emplois directs, indirects et induits ou bien encore l'implication d'une entreprise dans la vie locale (à travers la participation à des manifestations culturelles et/ou à des projets de développement) représentent autant d'outils de communication permettant de légitimer une activité au niveau local mais aussi au niveau européen (Couzinet et al., 2000).

Outre l'ancrage en termes d'image, nous avons vu plus haut comment l'ancrage productif était également susceptible d'évoluer au sein de l'interprofession roquefortaise, l'évolution de la demande a en effet eu un impact décisif sur l'évolution de l'aire de collecte. Dans un premier temps, les besoins croissants en lait ont provoqué un élargissement de la zone AOC. Puis, à partir des années 1970 , les progrès techniques ont contribué à une explosion de la productivité et ont amené les dirigeants de la filière à abandonner les bassins extérieurs et à se recentrer sur le sud du Massif Central, la demande n'étant plus en mesure de suivre l'offre.

Malgré les particularités des systèmes productifs bénéficiant d'une appellation, les produits AOC sont de plus en plus confrontés à la concurrence de marques commerciales (de distributeur ou non) faisant référence à des origines plus ou moins vagues. Cette évolution tend à relativiser la spécificité de l'appellation d'origine et oblige les acteurs des filières à s'engager davantage qu'auparavant dans des stratégies promotionnelles. Le lien entre politique de marketing et ancrage local mériterait à cet égard une analyse plus fournie: les AOC fromagères les plus récentes, nées dans un environnement concurrentiel plus prégnant et désormais international, sont-elles réellement moins ancrées à leur territoire? La recherche de rentabilité au détriment de coopérations locales - estelle aujourd'hui une préoccupation plus importante?

Enfin, les grandes et moyennes surfaces (GMS), qui contrôlent aujourd'hui la majorité de la distribution en France et en Europe par l'intermédiaire de centrales d'achat au pouvoir de négociation considérable, constituent pour les filières commercialisant des fromages sous $A O C$ un interlocuteur incontournable $^{19}$. Les exigences des distributeurs en matière de régularité et d'homogénéité des produits représentent pour les filières un ensemble de contraintes susceptibles d'avoir un impact sur l'organisation de la production et donc sur l'ancrage au niveau local.

- L'environnement institutionnel des filières fromagères d'AOC se manifeste, quant à lui, à diverses échelles géographiques.

Au niveau national, cet environnement correspond à une série d'organismes dotés chacun de missions et de compétences propres, parfois relayés à l'échelle régionale. D'une part, nous trouvons les organisations représentant l'ensemble de la profession laitière: principalement le Centre national interprofessionnel de l'industrie laitière (CNIEL), constitué des fédérations d'éleveurs, de coopératives et d'industriels laitiers, et l'Office national interprofessionnel du lait (ONILAIT), organisme de régulation du marché à caractère industriel et commercial. D'autre part, les organismes plus spécifiques aux AOC: l'Institut national des appellations

19. Le Roquefort, pour ne citer que lui, est commercialisé à $85 \%$ en GMS (chiffres 1997). 
d'origine (INAO), interlocuteur principal dans la procédure de reconnaissance du produit; l'Association nationale des appellations d'origine fromagères (ANAOF) et la Fédération nationale des appellations d'origine contrôlées fromagères (FNAOC), qui sont toutes deux des structures de défense des appellations fromagères.

L'ensemble de ces structures correspond à des échelles d'action très diverses (nationale, régionale, départementale...) dont les filières doivent tenir compte, notamment dans le cadre de leurs relations avec les pouvoirs publics (gestion des marchés, sécurité alimentaire...).

Les instances présentes à l'échelle internationale constituent, quant à elles, des facteurs contraignants, des remises en cause potentielles du concept même d'AOC. Au niveau européen, malgré des oppositions idéologiques encore fortes, la conception française de la qualité a servi de base aux réflexions relatives à la mise en place des AOP et des IGP en 1992. L'origine des produits fait en revanche l'objet de remises en cause beaucoup plus importantes dans le cadre des négociations commerciales au niveau de l'OMC. Considérées par beaucoup de pays comme des entraves au libéralisme, les AOC sont en permanence à la recherche d'arguments justifiant leurs spécificités. La suppression des quotas à l'échelle mondiale représente à cet égard une éventualité menaçant directement la politique de maîtrise de la production menée par les filières AOC depuis de nombreuses années.

Quoi qu'il en soit, ces sphères sociale, économique, politique et institutionnelle sont autant d'horizons stratégiques plus ou moins favorables selon les époques à l'ancrage territorial des filières et des acteurs qui les composent. Pour assurer leur reproduction, ces dernières doivent donc composer avec ces contraintes externes et s'adapter en permanence à ces contextes incertains.

\section{Conclusion}

La grille d'analyse ébauchée dans cet article s'inspire en grande partie du cas de l'interprofession roquefortaise, néanmoins son objectif est de s'appliquer à l'ensemble des $A O C$ fromagères, où les questions de mobilisation des ressources et de rapport au territoire des acteurs se posent avec autant d'acuité.

Au-delà de la vision en termes de localisation ou même de simple ressource, la notion d'ancrage telle qu'elle a été développée ici donne une autre illustration des relations complexes qui existent entre des acteurs économiques et leurs territoires. Dans le cas plus spécifique des entreprises commercialisant des produits sous une AOC, l'ancrage territorial est, selon les situations, un choix stratégique ou bien un point de passage obligé, qui implique la légitimité de l'acteur économique concerné au sein de la filière et au sein du territoire où il est inscrit (on pense bien sûr en premier lieu à la Société des Caves).

La réflexion menée sur Roquefort nous donne des éléments de réponse à propos du rôle joué par le territoire au sens large dans la capacité de reproduction et la performance ćconomique de la filic̀rc. À travers un ensemble de règles formelles et informelles, ainsi qu'au travers des relations privilégiées entre les acteurs, le système dans son ensemble est ainsi parvenu à résister aux évolutions extérieures et à conserver sa relative autonomie de fonctionnement.

Les indicateurs exposés ici relèvent certes de registres distincts, mais mériteraient d'être également considérés les uns par rapport aux autres, le tout dans une perspective historique: quelles sont les corrélations qui existent par exemple entre la stratégie de mobilisation des ressources d'une entreprise et son poids économique sur le territoire? Comment évaluer l'implication de cette entreprise dans la vie socio-économique locale? Sur quelles bases les politiques de promotion des produits sont-elles réalisées? 
Ainsi, la cohérence de l'articulation entre les trois formes d'ancrage que nous avons développées relève de la confrontation entre ces multiples indicateurs: peut-on faire apparaître une dynamique d'ensemble? Des trajectoires d'ancrage territorial spécifiques existent-elles? Et si oui, en fonction de quoi? La préservation et le développement de ces filières passent nécessairement à l'avenir par la prise en compte de l'ensemble de ces questionnements.

L'auteur tient à remercier Dominique Coquart et Valérie Olivier, respectivement professeur et maître de conférences en Économie à l'École nationale supérieure agronomique de Toulouse (Laboratoire Dynamiques Rurales), ainsi que Michel Roux, professeur de Géographie à l'Université de Toulouse-le-Mirail, pour leurs remarques constructives sur la première version de cet article. L'auteur reste bien entendu responsable des erreurs et omissions pouvant subsister dans cette version finale

\section{RÉFÉRENCES BIBLIOGRAPHIQUES}

Auriac F. Système économique et espace, le vignoble languedocien. Economica, collection Géographia, Paris, 1983, 215 p.

Benko G., Lipietz A. Les régions qui gagnent - les nouveaux paradigmes de la géographie économique. Presses Universitaires de France, collection Économie en Liberté, Paris, 1992, 424 p.

Bonniol J.-L., Saussol A. Grands Causses, nouveaux enjeux, nouveaux regards. Fédération pour la vie et la sauvegarde des Grands Causses, 1995, 416 p.

Brunet R., Ferras R., Théry H. Les mots de la géographie. Dictionnaire critique. Reclus. La Documentation française, Paris, 1993, coll. Dynamiques du Territoire, $518 \mathrm{p}$.

Chia E., Torre A. Règles et confiance dans un système localisé, le cas de la production de Comté AOC. Sciences de la Société, 1999, $n^{\circ} 48$, p. 49.

Couzinet L., Frayssignes J. Les filières de production alimentaire de qualité, stratégies de marché et développement rural - les exemples de la filière avicole label rouge Avigers et des filières fromagères AOC Rocamadour et Roquefort. Communication au colloque SFER-GEWISOLA «Agriculture et politique agricole en France et en Allemagne», Strasbourg, 2000, 16 p.

Couzinet L., Frayssignes J., Pouzenc M., Simonneaux J. The Enhancement of Rural Amenities, the Association of Individual Strategies and Collective Organization: the
Example of the Lot (France). Communication, 6th World Congress of Regional Science Association International: Firm strategies in a small world, 16-20 mai 2000, 29 p.

Frayssignes J. Le "système Roquefort»: un exemple d'évolution des relations entre une filière et son territoire. Mémoire de DEA, Université de Toulouse le Mirail, 1999, $95 \mathrm{p}$.

Guiraud S. Roquefort, un avenir à affiner. In Ressources, supplément au quotidien Midi Libre, 8 décembre, 1997, p. 1-4.

Perrier-Cornet Ph., Sylvander B. Firmes, coordination et territorialité, une lecture économique de la diversité des filières d'appellation d'origine. Économie Rurale, Paris, 2000, $\mathrm{n}^{\circ} 258$, p. 79-89.

Proulx M.-U. (dir.) Territoire et développement économique. L'Harmattan, Paris, coll. Villes et Entreprises, 1998, 314 p.

Ricard D. Les montagnes fromagères en France. Thèse de Doctorat, Université Blaise Pascal, Clermont-Ferrand, CERAMAC, 1993, 493 p.

Saglio J. Echange social et identité collective dans les systèmes industriels. Sociologie du Travail, 1991, n 4, p. 529.

Zimmermann J.-B. Nomadisme et ancrage territorial: propositions méthodologiques pour l'analyse des relations firmes-territoires. Revue d'Économie régionale et urbaine, 1998, n² 2 , p. 211-230. 